\title{
Clinical evaluation of QuantiFERON TB-2G test for immunocompromised patients
}

\author{
Y. Kobashi, K. Mouri, Y. Obase, M. Fukuda, N. Miyashita and M. Oka
}

ABSTRACT: The usefulness of the tuberculin skin test (TST) and the QuantiFERON TB-2G (QFTTB) test were compared in immunocompromised patients.

The subjects consisted of 252 immunocompromised patients who were clinically suspected of tuberculosis (TB) infection between April 2005 and December 2006.

Regarding the underlying diseases, 74 subjects had malignant diseases, 72 were undergoing immunosuppressive treatment, $\mathbf{5 2}$ had diabetes mellitus, $\mathbf{5 0}$ had chronic renal failure and four had HIV infection. While the positive rate of the QFT-TB test for the diagnosis of TB infection (TB

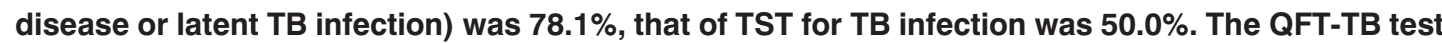
was significantly better than TST. However, $32(13 \%)$ patients had an indeterminate QFT-TB result. Indeterminate findings were significantly more frequent in patients receiving immunosuppressive treatment $(28 \%)$, especially with lymphocytopaenia in the peripheral blood, than in those who had other underlying diseases. While TST-positive and QFT-TB test-negative results were recognised in immunocompromised patients with bacille Calmette-Guérin vaccination or nontuberculous mycobacterial disease, TST-negative and QFT-TB test-positive results were recognised in immunocompromised patients with a past history of TB infection.

It was concluded that the QuantiFERON TB-2G test is a more useful diagnostic method for tuberculosis infection than tuberculin skin test for immunocompromised patients suspected of tuberculosis disease. However, because the results of the QuantiFERON TB-2G test show an indeterminate response for patients receiving immunosuppressive treatment, especially for those with lymphocytopaenia due to severe underlying diseases, care must be taken in the interpretation of the QuantiFERON TB-2G test for these patients.

KEYWORDS: Immunocompromised patient, QuantiFERON TB-2G, tuberculin skin test

I

n Japan, the incidence of tuberculosis (TB) is intermediate (35 cases per 100,000 population) and has recently been decreasing. However, aging of the population and the increased use of immunosuppressive treatments (e.g. cancer chemotherapy and immunomodulatory agents) highlight the need for additional strategies to maintain and improve TB control [1]. Early diagnosis of infectious cases and treatment of these immunocompromised patients infected with Mycobacterium tuberculosis (MTB) are important strategies for reducing the incidence of TB in industrialised countries $[1,2]$. The specificity of the tuberculin skin test (TST) is limited by crossreactivity of the purified protein derivative (PPD) with bacille Calmette-Guérin (BCG) vaccine and with most nontuberculous mycobacteria (NTM) [3]. Its sensitivity is also low in immunocompromised patients, in whom the risk of progression to TB is high [2]. Despite these limitations, TST is routinely used in hospital clinical practice to screen for latent TB infection [4].
The two commercialised ex vivo interferon (IFN)$\gamma$ assays, QuantiFERON TB-2G (QFT-TB; Cellestis Ltd, Carnegie, Victoria, Australia) and TSPOT.TB (Oxford Immunotec, Oxford, UK), use early secretory antigenic target (ESAT)-6 and culture filtrate protein (CFP)-10 as MTB-specific stimulants on ELISA and enzyme-linked immunospot assay (ELISPOT), respectively [5-8]. The US Food and Drug Administration has approved the QFT-TB test and is evaluating the T-SPOT.TB test, which has been approved for use in Europe. These tests demonstrate a positive result for most individuals with a high likelihood of TB infection (TB disease or latent TB infection) and a negative result for BCG-vaccinated individuals with a low likelihood of TB infection. Of these tests, the QFTTB was first used commercially in Japan in April 2005 for the diagnosis of TB infection. Although there have been several reports investigating whether QFT-TB test and T-SPOT.TB test would be useful in immunocompromised patients [9$11]$, there has been only one report investigating
AFFILIATIONS

Division of Respiratory Diseases, Dept of Medicine, Kawasaki Medical School, Kurashiki, Japan.

CORRESPONDENCE

Y. Kobashi

Division of Respiratory Diseases Dept of Medicine

Kawasaki Medical School

577 Matsushima

Kurashiki

701-0192

Japan

Fax: 81864641041

E-mail: yoshihiro@med.kawasakim.ac.jp

Received:

April 042007

Accepted after revision:

July 162007

STATEMENT OF INTEREST

None declared. 
the usefulness of the new test separately in individual important underlying disease [11]. Therefore, the present authors prospectively report on the results of an analysis in which the QFT-TB test was routinely performed on many consecutive immunocompromised patients separated according to individual underlying disease in several community hospitals. The present study evaluated the feasibility and performance of the QFT-TB test compared with those of TST for immunocompromised patients in a hospital-based population, and included the interpretation of concordant and discordant results of both tests.

\section{METHODS}

\section{Study population}

The study was approved by the Ethics Committee of Kawasaki Medical School (Kurashiki, Japan). In total, 264 immunocompromised patients ( $>16$ yrs old) with underlying diseases who were clinically suspected of TB disease were prospectively enrolled in the present study between April 2005 and December 2006. The patients suspected of TB disease demonstrated the appearance of new lesions in the lung field, pleural effusion, or lymphadenopathy on chest radiograph or chest computed tomography (CT) during follow-up of the underlying disease. Of these, 12 patients were excluded because there were no obvious findings suggesting TB disease on chest CT. The remaining 252 immunocompromised patients (204 inpatients and 48 outpatients) were analysed in the present study. Most of these patients had the underlying disease on admission for which they consulted the following hospitals: Kawasaki Medical School Hospital, Kawasaki Medical School Kawasaki Hospital, Kurashiki Central Hospital, Kurashiki Daiichi Hospital (all Kurashiki, Japan), and Asahigaoka Hospital (Okayama, Japan). Written, informed consent was obtained from all participants in the present study. All patients except for the four with HIV infection had negative results on serological tests for HIV or had no obvious risk factors for HIV infection. Demographic, clinical, radiological and microbiological data were collected for all patients. Collected data included any history of previous TB disease and risk factors for TB (i.e. malignant diseases, including leukaemia; immunosuppressive treatments, such as systemic immunosuppressive drugs or anti-tumour necrosis factor (TNF)- $\alpha$ agents within the past 3 months; diabetes mellitus; chronic renal failure with haemodialysis; and HIV infection with anti-HIV treatment). The diagnosis of TB disease was definitively confirmed by culture of sputum, bronchoalveolar lavage fluid (BALF) or pleural fluid samples found to be positive for MTB microbiologically. In Japan, inoculation with the BCG vaccination is first administered during infancy. Thereafter, if the response to TST is negative at the time of entrance of junior high school, BCG vaccination is administered again until conversion of the TST to positive. Information regarding any previous Mantoux TST results and BCG vaccination, as well as information about clinical and laboratory findings, was collected from each patient at the time of enrolment. Sputum or other appropriate respiratory samples were collected from all patients, and culture samples were obtained for the detection of MTB.

\section{Sample collection and TST}

A heparinised blood sample was collected from individual patients by vein puncture for whole-blood IFN- $\gamma$ assay. Blood samples were collected before administration of the Mantoux TST. For the TST, $0.1 \mathrm{~mL}$ of tuberculin PPD (Nippon BCG, Tokyo, Japan; equivalent to three tuberculin units of PPDsaline) was injected intradermally into the volar aspect of the forearm, and the transverse induration diameter was measured $48 \mathrm{~h}$ later. The TST results were interpreted according to the level of risk, as reported in current guidelines [12].

\section{QuantiFERON TB-2G test}

The QFT-TB test was performed according to the recommendations of the manufacturer (Cellestis Ltd). Briefly, the test consisted of a negative control (a "nil" well, i.e. whole blood without antigens or mitogen), a positive control (a "mitogen" well, i.e. whole blood stimulated with the mitogen phytohaemaggulutinin) and two "sample" wells (i.e. whole blood stimulated with either ESAT-6 or CFP-10). Whole-blood specimens were incubated for $18 \mathrm{~h}$ (overnight) at $37^{\circ} \mathrm{C}$ in a humidified atmosphere. The IFN- $\gamma$ level of the nil well was considered to be the background value and was subtracted from the values for the mitogen well and the antigenstimulated wells. The test result was considered positive and suspected of TB infection if the IFN- $\gamma$ level in the sample well after stimulation with ESAT-6 and/or CFP-10 was $>0.35 \mathrm{IU} \cdot \mathrm{mL}^{-1}$ (after subtraction of the value for the nil well), irrespective of the result for the positive control well. The test result was considered negative and it was difficult to diagnose TB infection if the IFN- $\gamma$ level was $<0.35 \mathrm{IU} \cdot \mathrm{mL}^{-1}$ and if the IFN- $\gamma$ level of the positive control well (after subtraction of the value for the nil well) was $>0.5 \mathrm{IU} \cdot \mathrm{mL}^{-1}$. The test result was considered indeterminate and it was impossible to interpret the result if the IFN- $\gamma$ level was $<0.35 \mathrm{IU} \cdot \mathrm{mL}^{-1}$ in both antigen wells and $<0.5 \mathrm{IU} \cdot \mathrm{mL}^{-1}$ in the positive control well, or if the IFN- $\gamma$ level was below half of the negative control well in both antigen wells and $>0.7 \mathrm{IU} \cdot \mathrm{mL}^{-1}$ in the negative control well. This judgment was performed according to the guidelines proposed by the Centers for Disease Control and Prevention for using the QFT-TB test [13].

\section{Statistical analysis}

Information from the questionnaires, TST results and wholeblood IFN- $\gamma$ assay results were subjected to statistical analyses. Statistical analyses were performed to assess the following: 1) the feasibility and performance of the QFT-TB test compared with those of TST; 2) the proportion of QFT-TB tests with an indeterminate result and the associated risk factors; 3) the concordance and discordance between the QFT-TB and TST results; and 4) the positive rate of the QFT-TB and TST results in patients with a final diagnosis of active TB disease. The analysis of concordance between the QFT-TB and TST results was calculated using the $\kappa$-value. Both the QFT-TB and TST results were compared using the Chi-squared test. The Wilson score method was used to calculate $95 \%$ confidence intervals for the positive rate of both tests [14].

\section{RESULTS}

In total, 252 patients who were clinically suspected of TB disease were tested with the TST and the QFT-TB test between April 2005 and December 2006. Their demographic and clinical characteristics are shown in table 1 . Regarding the underlying diseases among these patients, 74 patients had malignant diseases (all of these patients had advanced cancer without 
surgical treatment), including leukaemia (12 patients during the follow-up period including patients receiving anti-cancer therapy); 72 were undergoing immunosuppressive treatment for underlying diseases (52 were receiving the systemic steroid prednisone, 10 were receiving anti-TNF- $\alpha$ agents and 10 were receiving the systemic steroid prednisone plus other immunosuppressive agents); 52 had diabetes mellitus (all patients were receiving oral hypoglycaemic agents or insulin); 50 had chronic renal failure (all patients were receiving haemodialysis); and four had HIV infection (all patients were receiving anti-HIV treatment). The mean age of all immunocompromised patients was 62.0 yrs and there were 156 males and 96 females. A past history of BCG vaccination was present in 152 (60.3\%) patients and $24(9.5 \%)$ had a past history of healed pulmonary TB. The incidence of TB disease was $12.7 \%$ in the overall patient group. On separation of patients by underlying immunocompromised diseases, there were no significant differences, but in laboratory findings, the peripheral lymphocyte count and CD4 lymphocyte count were significantly lower in patients with immunosuppressive treatment than in those with other underlying diseases. There were $31(12.3 \%)$ patients with bed-ridden status and there were no significant differences between individual groups with any underlying disease.

Among the 252 immunocompromised patients who underwent the QFT-TB test, 32 (12.7\%) patients had an indeterminate result. All of these 32 patients showed a positive-control failure of the QFT-TB result $\left(\mathrm{IFN}-\gamma<0.5 \mathrm{IU} \cdot \mathrm{mL}^{-1}\right)$. On univariate analysis (not shown in the present article), patients undergoing immunosuppressive treatments had the highest and most significant proportion of indeterminate QFT-TB results (odds ratio $(\mathrm{OR}) 3.64 ; \mathrm{p}=0.0008)$ compared with other patients with immunocompromised diseases (malignant diseases: OR 2.28, $p=0.201$; diabetes mellitus: OR $1.38, p=0.780$; chronic renal failure: OR 1.45, $\mathrm{p}=0.695$; and HIV infection). On multivariate analysis (table 2), patients undergoing immunosuppressive treatments also had similar and the highest and most significant proportion of indeterminate QFT-TB results. The QFT-TB test produced a significantly higher proportion of indeterminate results in patients with a negative TST result (24 out of $150 ; 16.0 \%)$, compared with that in TST-positive patients (eight out of $102 ; 7.8 \% ; \mathrm{p}<0.05$ ). The distribution between the TST results and the QFT-TB test results in all immunocompromised patients is shown in table 3. Indeterminate QFT-TB test results were significantly more frequent in patients with a TST-negative result than in those with a TST-positive result $(\mathrm{p}<0.05)$.

Among all patients tested with the QFT-TB test and the TST, 32 $(12.7 \%)$ were diagnosed with TB disease because they were culture-positive for MTB. The QFT-TB test and TST results of these patients are shown in table 4 . The positive response rate for the QFT-TB test of 32 patients with TB disease (25 out of 32; $78.1 \%$ ) was significantly higher than that for TST (16 out of 32; $50.0 \% ; \mathrm{p}<0.05)$.

The concordance rate between the QFT-TB test and the TST was $59.5 \%$ (150 out of 252) with a p-value of $0.56(95 \%$ confidence interval $0.32-0.68)$. In total, 64 patients (25.4\%) had a positive TST result and a negative QFT-TB test result; of those, $52(81.3 \%)$ were BCG vaccinated, $10(15.6 \%)$ had a NTM disease, and the remaining two were unknown. Six $(2.4 \%)$

TABLE 1 Clinical characteristics and laboratory findings of immunocompromised patients who were suspected of tuberculosis (TB) infection

Characteristics

Underlying disease

Total

\begin{tabular}{ccccc}
\hline Malignant disease $^{\#}$ & $\begin{array}{c}\text { Immunosuppressive } \\
\text { treatment }\end{array}$ & $\begin{array}{c}\text { Diabetes } \\
\text { mellitus }\end{array}$ & $\begin{array}{c}\text { Chronic renal } \\
\text { failure }\end{array}$ & HIV infection
\end{tabular}

\begin{tabular}{|c|c|c|c|c|c|c|}
\hline Subjects n & 74 & 72 & 52 & 50 & 4 & 252 \\
\hline Age yrs & $63.4 \pm 10.8$ & $60.5 \pm 10.4$ & $68.2 \pm 11.2$ & $64.6 \pm 10.8$ & 42.0 & $62.0 \pm 10.4$ \\
\hline Male/female $\mathbf{n}$ & $48 / 26$ & $37 / 35$ & $35 / 17$ & $32 / 18$ & $4 / 0$ & $156 / 96$ \\
\hline Smoker & $50(67.6)$ & $43(59.7)$ & $36(69.2)$ & $33(66.0)$ & $3(75.0)$ & $165(65.5)$ \\
\hline Alcohol abuse & $13(17.6)$ & $9(12.5)$ & $10(19.2)$ & $8(16.0)$ & 0 & $40(15.9)$ \\
\hline Past history of TB & $7(9.5)$ & $5(6.9)$ & $6(11.5)$ & $6(12.0)$ & 0 & $24(9.5)$ \\
\hline Bed-ridden status & $9(12.2)$ & $9(12.5)$ & $6(11.5)$ & $6(12.0)$ & $1(25.0)$ & 31 (12.3) \\
\hline BCG vaccination & $45(60.8)$ & $43(59.7)$ & $31(59.6)$ & $30(60.0)$ & $3(75.0)$ & $152(60.3)$ \\
\hline WBC count cells $\mu \mathrm{L}^{-1}$ & $3476 \pm 485$ & $2890 \pm 564$ & $3762 \pm 570$ & $3702 \pm 546$ & 2960 & $3480 \pm 504$ \\
\hline Lymphocyte count cells $\mu \mathrm{L}^{-1}$ & $502 \pm 52$ & $380 \pm 36^{*}$ & $576 \pm 58$ & $522 \pm 54$ & 296 & $496 \pm 50$ \\
\hline CD4 lymphocyte cells $\cdot \mu \mathrm{L}^{-1}$ & $208 \pm 31$ & $114 \pm 29 *$ & $220 \pm 36$ & $212 \pm 32$ & 40 & $196 \pm 32$ \\
\hline Total protein $\mathbf{g} \cdot \mathbf{d L}^{-1}$ & $6.4 \pm 1.4$ & $6.6 \pm 1.6$ & $6.8 \pm 1.5$ & $6.8 \pm 1.5$ & 6.8 & $6.7 \pm 1.5$ \\
\hline Alb $\mathbf{g} \cdot \mathbf{d L}^{-1}$ & $3.3 \pm 0.7$ & $3.5 \pm 0.8$ & $3.6 \pm 0.8$ & $3.6 \pm 0.8$ & 3.6 & $3.5 \pm 0.8$ \\
\hline r-Glb g.dL ${ }^{-1}$ & $1.1 \pm 0.3$ & $1.4 \pm 0.4$ & $1.2 \pm 0.3$ & $1.3 \pm 0.3$ & 1.4 & $1.2 \pm 0.3$ \\
\hline TB disease & $10(13.5)$ & $9(12.5)$ & $7(13.5)$ & $6(12.0)$ & 0 & $32(12.7)$ \\
\hline
\end{tabular}

Data are presented as mean \pm SD or $n(\%)$, unless otherwise indicated. BCG: bacille Calmette-Guérin; WBC: white blood cell; Alb: albumin; r-Glb: r-globurin. ${ }^{\#}$ : patients with a diagnosis of malignant disease who were receiving anti-cancer therapy $(n=24)$ and who were not receiving anti-cancer therapy ( $n=50$; including 12 patients with leukaemia). $"$ : patients receiving the systemic steroid prednisone $(n=52)$ or anti-tumour necrosis factor- $\alpha$ agents ( $n=10)$ at the time of testing with QuantiFERON TB-2G, or those receiving prednisone plus other immunosuppressive drugs. *: $p<0.05$. 
TABLE 2 Multivariate analysis of the indeterminate QuantiFERON TB-2G (QFT-TB) results

\begin{tabular}{|c|c|c|c|c|c|}
\hline Underlying disease & Subjects $n$ & Indeterminate ${ }^{\#}$ & Determinate & OR $(95 \% \mathrm{Cl})$ & p-value \\
\hline Malignant disease & 74 & $8(10.8)$ & $66(89.2)$ & $2.19(0.75-4.88)$ & 0.201 \\
\hline Immunosuppressive treatment ${ }^{+}$ & 72 & $20(27.8)$ & $52(72.2)$ & $3.80(2.10-9.54)$ & 0.0006 \\
\hline Diabetes mellitus & 52 & $2(3.8)$ & 50 (96.2) & $1.48(0.65-2.60)$ & 0.780 \\
\hline Total & 252 & $32(12.7)$ & $220(87.3)$ & & \\
\hline
\end{tabular}

Data are presented as $\mathrm{n}(\%)$, unless otherwise indicated. OR: odds ratio; Cl: confidence interval; ND: not determined. ${ }^{\#}$ : all indeterminate QFT-TB results were due to a low response to the mitogen in the positive control well, according to the manufacturer's instructions. " : patients with a diagnosis of malignant disease who were receiving anti-cancer therapy $(n=18)$ and who were not receiving anti-cancer therapy $(n=56){ }^{+}$: patients receiving the systemic steroid prednisone $(n=52)$ or anti-tumour necrosis factor- $\alpha$ agents $(n=10)$ at the time of testing with QFT-TB, or prednisone plus other immunosuppressive drugs $(n=10)$

patients had a positive QFT-TB result and a negative TST result. Although none of these patients had been BCG vaccinated, five out of six had a past history of TB disease and one was unknown. Out of 252 patients, 152 (60.3\%) on whom both tests were performed had been BCG vaccinated. In this subgroup, TST was positive in 96 (63.2\%) and QFT-TB test was positive in $30(19.7 \% ; \mathrm{p}<0.05)$. The concordance rate between the TST and the QFT-TB test was significantly lower among BCG-vaccinated individuals than among non-BCGvaccinated subjects $(27.8$ versus $62.0 \% ; \mathrm{p}<0.05)$.

Five (15.6\%) out of 32 patients with a QFT-TB indeterminate response appeared to have TB disease; 25 (69.4\%) out of 36 patients with a QFT-TB-positive response appeared to have TB disease. Two patients out of 184 with a QFT-TB-negative response appeared to have TB disease. While 16 (15.7\%) patients appeared to have TB disease out of 102 with a TST-positive response, $16(10.7 \%)$ patients among 150 with a TST-negative response also appeared to have TB disease. The incidence of TB disease was significantly higher in the QFT-TB-positive response group than in TST-positive response group $(\mathrm{p}<0.05)$.

\section{DISCUSSION}

In Japan, most patients receiving immunosuppressive treatment for systemic underlying diseases have a past history of

\begin{tabular}{lccc} 
TABLE 3 & $\begin{array}{l}\text { QuantiFERON TB-2G } \\
\text { skin test (TST) } \\
\text { patients }\end{array}$ & results for immunocompromised \\
QFT-TB & \multicolumn{3}{c}{ TST } \\
\cline { 2 - 4 } & Positive & Negative & Total \\
\hline Positive & 30 & 6 & 36 \\
Negative & 64 & 120 & 184 \\
Indeterminate & 8 & 24 & 32 \\
Total & 102 & 150 & 252 \\
\hline & & & \\
Data are presented as $\mathrm{n}$. &
\end{tabular}

BCG vaccination. However, it has been noted that the sensitivity of TST is not high in immunosuppressed patients, in whom the risk of progression to TB is high [3]. In place of the TST test in vivo, the QFT-TB test in vitro was first used commercially for patients with TB infection in Japan in April 2005 because the QFT-TB test demonstrated a negative result for BCG-vaccinated individuals and a negative result for most NTM [2, 15]. Therefore, the present authors prospectively investigated the results of QFT-TB test being routinely performed on consecutive immunocompromised patients in several community hospitals. Although the patients were separated according to individual systemic underlying diseases (malignant diseases including leukaemia, those receiving immunosuppressive treatment, diabetes mellitus, chronic renal failure and HIV infection) that induce immunosuppression, there were no significant differences among the clinical characteristics (table 1). However, while no significant differences could be detected between other immunosuppressive patients in the laboratory findings, except for those in several patients with HIV infection, patients receiving immunosuppressive treatment showed significantly lower lymphocyte and CD4 lymphocyte counts than other immunocompromised patients.

Regarding the specificity and sensitivity of the QFT-TB test, MORI et al. [6] reported a sensitivity of $89 \%$ in a selected

\begin{tabular}{lccc} 
TABLE 4 & $\begin{array}{l}\text { QuantiFERON TB-2G } \\
\text { skin test (TST) results for immunocompromised } \\
\text { patients with tuberculosis (TB) disease }\end{array}$ \\
\cline { 2 - 4 } QFT-TB & \multicolumn{3}{c}{ TST } \\
\cline { 2 - 4 } & Positive & Negative & Total \\
\hline Positive & 15 & 10 & 25 \\
Negative & 0 & 2 & 2 \\
Indeterminate & 1 & 4 & 5 \\
Total & 16 & 16 & 32 \\
\hline & & & \\
Data are presented as $\mathrm{n}$. & &
\end{tabular}


population of patients with clinical signs suggestive of TB infection. They also reported a specificity of $98 \%$ in low-risk subjects who had been vaccinated with BCG and who were assumed to be truly free of TB. However, in the present study, the positive response rate for the QFT-TB test was relatively low at $78.1 \%$ ( 25 out of 32 patients with TB disease). An indeterminate result for the QFT-TB test was recognised in $12.7 \%$ (32 out of 252 immunocompromised patients) and it appeared most frequently in patients receiving immunosuppressive treatments who presented with lymphocytopaenia (especially CD4 lymphocytopaenia). In previous reports, FERRARA et al. [9] noted that the QFT-TB-positive control failed in $21 \%$ of tests performed in routine clinical diagnostic microbiology laboratories and community-based contact tracing protocols, and that these indeterminate results were significantly overrepresented among patients receiving immunosuppressive treatments. A similar result (indeterminate rate: $11 \%$ ) has been confirmed in a prospective study of 393 individuals [16]; however, there was no speculation concerning the reason. In the present study, a similar result was obtained and all patients showed QFT-TB-positive control failure (IFN- $\gamma$ $\left.<0.5 \mathrm{IU} \cdot \mathrm{mL}^{-1}\right)$, although the indeterminate result of the QFTTB test was $12.7 \%$, similar to the data reported by FERRARA et al. [9]. One reason was that lymphocytopaenia, especially CD4 lymphocytopaenia, predominantly appeared in patients with immunosuppressive treatment (table 1) [17]. The QFT-TB test depends on the elaboration of inflammatory cytokines by $\mathrm{T}$ cells previously sensitised to MTB-specific antigens. In the blood, mononuclear cells from peripheral blood are stimulated in vitro, and the production of IFN- $\gamma$ from sensitised Tlymphocytes by MTB-specific antigen is measured by ELISA in the QFT-TB test [18]. Therefore, it was first speculated that lymphocytopaenia caused decreased production of IFN- $\gamma$ and lower mitogen, ESAT-6 or CFP-10 QFT-TB levels. Secondly, immunosuppressive drugs, such as corticosteroid drugs, directly reduce the production of inflammatory cytokines, such as IFN- $\gamma$, interleukin- 1 and TNF- $\alpha$ from T-lymphocytes $[13,19,20]$. Finally, the decrease of IFN- $\gamma$ induced indeterminate results of the QFT-TB test because of the lower mitogen, ESAT-6 or CFP-10 QFT-TB levels. For these reasons, indeterminate results due to positive control failure seem to be less frequent with the T-SPOT.TB test, which detects individual Tcells producing IFN- $\gamma$ using an ELISPOT method, than the QFT-TB test $[10,16,21]$. This also applies to the use of the TSPOT.TB in subgroups with impaired cellular immunity, with a recent report that HIV-infected subjects tested with the TSPOT.TB showed only one (3\%) indeterminate result, and that positive control responses were not adversely affected by CD4 counts [22]. Otherwise, a new IFN- $\gamma$ release assay including MTB-specific antigen (TB 7.7; QuantiFERON TB-Gold In-Tube; Cellestis Ltd) [23] has recently been developed and will replace the QFT-TB-2G test in Japan as soon as possible. Immunocompromised patients suspected of TB infection had to be examined using the QFT-TB test in the present study because only this test is currently commercially available in Japan. Hereafter, although LiEBESCHUETz et al. [24] had reported the results of ELISPOT test for immunosuppressed populations with HIV and suspected TB disease, the present authors would like to carry out a prospective, large-scale study of immunocompromised patients with various severe underlying diseases other than HIV infection in a community hospital-based population with suspected TB infection using the T-SPOT.TB test instead of the QFT-TB test.

Regarding the comparison of TST and QFT-TB test results, the present findings support the conclusion that QFT-TB test provides more accurate results than TST in immunocompromised patients [25]. The findings also suggest that the QFT-TB test might have limited clinical usefulness in patients receiving immunosuppressive treatment. However, in most patients, the QFT-TB test produced a valid result, although this IFN- $\gamma$ blood test may raise some questions regarding the validity of a negative TST result. Specifically, because TST does not have an internal positive control the clinician cannot distinguish between a true-negative result and a false-negative result. With the QFT$\mathrm{TB}$ test, a proportion of false-negative test results will be scored as indeterminate, allowing the clinician to disregard such results. Naturally, there would still be a certain proportion of falsenegative test results associated with a valid positive control, as was also observed. The present authors think that the reasons for the discordances between the TST and QFT-TB tests can be predominantly explained by BCG vaccination or NTM infection causing the result of TST to be positive and that of QFT-TB test to be negative, and by a past history of TB infection causing the result of TST to be negative and that of QFT-TB test to be positive, based on the results of the present study.

In conclusion, the present study demonstrated the clinical utility of the QuantiFERON TB-2G test compared with the tuberculin skin test for immunocompromised patients with tuberculosis infection. However, because the QuantiFERON TB-2G test result showed an indeterminate response for patients receiving immunosuppressive treatment, especially with lymphocytopaenia due to severe underlying diseases, care must be taken when making a diagnosis of tuberculosis for these patients based on QuantiFERON TB-2G test results. In the future, the present authors would like to increase the diagnostic rate of tuberculosis infection (tuberculosis disease or latent tuberculosis infection) for immunocompromised patients with underlying diseases in a community hospitalbased population suspected of tuberculosis infection by utilising other interferon- $\gamma$ detecting methods (T-SPOT.TB or QuantiFERON-TB Gold In-Tube).

\section{ACKNOWLEDGEMENTS}

The authors would like to thank T. Matsushima (Kurashiki Daiichi Hospital, Kurashiki, Japan; Asahigaoka Hospital, Okayama, Japan), N. Okimoto (Kawasaki Medical School Kawasaki Hospital, Kurashiki, Japan) and T. Kageoka (Kurashiki Central Hospital, Kurashiki, Japan) for helpful comments.

\section{REFERENCES}

1 Horsburgh CR Jr. Priorities for the treatment of latent tuberculosis infection in the United States. N Engl J Med 2004; 350: 2060-2067.

2 Advisory Council for the Elimination of Tuberculosis (ACET). Tuberculosis elimination revisited: obstacles, opportunities, and a renewed commitment. MMWR Recomm Rep 1999; 48: 1-13.

3 Heubner RE, Schein MF, Bass JB Jr. The tuberculin skin test. Clin Infect Dis 1993; 17: 968-975. 
4 American Thoracic Society and the Centers for Disease Control and Prevention. Diagnostic standards and classification of tuberculosis in adults and children. Am J Respir Crit Care Med 2000; 161: 1376-1395.

5 Brock I, Weldingh K, Lillebaek T, Follmann F, Andersen P. Comparison of tuberculin skin test and new specific blood test in tuberculosis contacts. Am J Respir Crit Care Med 2004; 170: 65-69.

6 Mori T, Sakatani M, Yamagishi F, et al. Specific detection of tuberculosis infection: an interferon-gamma-based assay using new antigens. Am J Respir Crit Care Med 2004; 170: 59-64.

7 Lalvani A, Nagvenkar P, Udwadia Z, et al. Enumeration of $\mathrm{T}$ cells specific for RD1-encoded antigen suggests a high prevalence of latent Mycobacterium tuberculosis infection in healthy urban Indians. J Infect Dis 2001; 183: 469-477.

8 Lalvani A, Pathan AA, McShane H, et al. Rapid detection of Mycobacterium tuberculosis infection by enumeration of antigen-specific cells. Am J Respir Crit Care Med 2001; 163: 824-828.

9 Ferrara G, Losi M, Meacci M, et al. Routine hospital use of a new commercial whole blood interferon- $\gamma$ assay for the diagnosis of tuberculosis infection. Am J Respir Crit Care Med 2005; 172: 631-635.

10 Lee JY, Choi HJ, Park IN, et al. Comparison of two commercial interferon- $\gamma$ assays for diagnosing Mycobacterium tuberculosis infection. Eur Respir J 2006; 28: 24-30.

11 Rangaka MX, Wilkinson KA, Seldon R, et al. Effect of HIV-1 infection on T-cell-based and skin test detection of tuberculosis infection. Am J Respir Crit Care Med 2007; 175: 514-520.

12 American Thoracic Society. Targeted tuberculin testing and treatment of latent tuberculosis infection. MMWR Recomm Rep 2000; 49: 1-51.

13 Mazurek GH, Jereb J, Lobue P, et al. Guidelines for using the QuantiFERON-TB Gold test for detecting Mycobacterium tuberculosis infection, United States. MMWR Recomm Rep 2005; 54: 49-55.

14 Newcombe RG. Two-sided confidence intervals for the single proportion: comparison of seven methods. Stat Med 1998; 17: 857-872.
15 Kobashi Y, Obase Y, Fukuda M, Yoshida K, Miyashita N, Oka M. Clinical reevaluation of the QuantiFERON TB-2G test as a diagnostic method for differentiating active tuberculosis from nontuberculous mycobacteriosis. Clin Infect Dis 2006; 43: 1540-1546.

16 Ferrara G, Losi M, D'Amico R, et al. Use in routine clinical practice of two commercial blood tests for diagnosis of infection with Mycobacterium tuberculosis: a prospective study. Lancet 2006; 367: 1328-1334.

17 Stuck AE, Minder CE, Frey FJ. Risk of infection complication in patients taking glucocorticosteroids. Rev Infect Dis 1989; 11: 954-963.

18 Andersen P, Munk ME, Pollock JM, Doherty TM. Specific immune-based diagnosis of tuberculosis. Lancet 2000; 256: 1099-1104.

19 Guyre PM, Girard MT, Morganelli PM, Manganiello PD. Glucocorticoid effects on the production and actions of immune cytokines. J Steroid Biochem 1988; 30: 89-93.

20 Brack A, Rittner HL, Younge BR, Kaltschmidt C, Weyand CM, Gorozny JJ. Glucocorticoid-mediated repression of cytokine gene transcription in human arteritis-SCID chimeras. J Clin Invest 1997; 99: 2842-2850.

21 Meier T, Eulenbruch HP, Wrighton-Smith P, Enders G, Regnath T. Sensitivity of a new commercial enzyme-linked immunospot assay (T SPOT-TB) for diagnosis of tuberculosis in clinical practice. Eur J Clin Microbiol Infect Dis 2005; 24: 529-536.

22 Dheda K, Lalvani A, Miller RF, et al. Performance of a Tcell-based diagnostic test for tuberculosis infection in HIVinfected individuals is independent of CD4 cell count. AIDS 2005; 19: 2038-2041.

23 Pai M, Joshi R, Dogra S, et al. Serial testing of health care workers for tuberculosis using interferon- $\gamma$ assay. Am J Respir Crit Care Med 2006; 174: 349-355.

24 Liebeschuetz S, Bamber S, Ewer K, Deeks J, Pathan AA, Lalvani A. Diagnosis of tuberculosis in South African children with a $\mathrm{T}$ cell-based assay: a prospective cohort study. Lancet 2004; 364: 2196-2203.

25 Richeldi L. An update on the diagnosis of tuberculosis infection. Am J Respir Crit Care Med 2006; 174: 736-742. 